\title{
TTR
}

Traduction, terminologie, re?daction

\section{Vernacularisation et traduction des textes pragmatiques en Afrique}

\section{Raymond Mopoho}

Volume 10, numéro 1, 1er semestre 1997

Langues, traduction et post-colonialisme

Languages, Translation and Post-Colonialism

URI : https://id.erudit.org/iderudit/037286ar

DOI : https://doi.org/10.7202/037286ar

Aller au sommaire du numéro

\section{Éditeur(s)}

Association canadienne de traductologie

ISSN

0835-8443 (imprimé)

1708-2188 (numérique)

Découvrir la revue

Citer cet article

Mopoho, R. (1997). Vernacularisation et traduction des textes pragmatiques en Afrique. TTR, 10(1), 245-261. https://doi.org/10.7202/037286ar
Résumé de l'article

Vernacularisation et traduction des textes pragmatiques en Afrique - La traduction des textes comportant des lacunes d'ordre grammatical, lexical, stylistique ou idiomatique présente habituellement des difficultés particulières, lesquelles sont amplifiées lorsqu'elles sont attribuables à la vernacularisation d'une langue étrangère. Dans les sociétés postcoloniales, l'absence ou la non-disponibilité des études linguistiques sur la plupart des langues locales rend ardue l'analyse des interférences entre ces dernières et les langues officielles étrangères. Cette situation, ajoutée à la grande diversité ethnolinguistique ambiante, ne facilite pas l'interprétation des textes produits par les personnes semi-lettrées. Le traducteur de ces textes se présente davantage comme un rédacteur qui, à partir de l'idée globale qui se dégage de l'original, conçoit et produit un texte répondant aux normes de la langue cible. L'évaluation d'un tel travail ne peut se faire qu'en comparant la finalité des deux textes.
Tous droits réservés (C TTR: traduction, terminologie, rédaction — Les auteurs, 1997
Cedocument est protégé par la loi sur le droit d'auteur. L'utilisation des services d'Érudit (y compris la reproduction) est assujettie à sa politique d'utilisation que vous pouvez consulter en ligne.

https://apropos.erudit.org/fr/usagers/politique-dutilisation/ 


\section{Vernacularisation et traduction des textes pragmatiques en Afrique}

\section{Raymond Mopoho}

La notion de vernacularisation renvoie au fait, pour des populations autochtones, de s'approprier une langue étrangère en la modifiant afin de la rendre capable d'exprimer leurs umanières de penser et de concevoipo (Manessy, 1984). Cette notion est empruntée à la théorie de la pidginisation, où elle décrit un processus intermédiaire qui suit l'indigénisation (la nativization de Hall, 1966) et précède la créolisation proprement dite. La vernacularisation, comme la plupart des phénomènes qui affectent la langue, suscite généralement des réactions divergentes ${ }^{l}$ : les protagonistes de l'expansion de la langue étrangère la saluent comme un pas de plus vers la consolidation de son statut international, les puristes y voient une menace pour la survie de la langue dans sa abonne formex, et les autochtones nationalistes dénoncent en elle la manifestation d'un impérialisme linguistique. Quoi qu'il en soit, la vernacularisation résulte souvent de l'expansion du rôle de la langue étrangère aux dépens de celui des langues locales. Or, cette expansion n'est pas toujours suivie d'un accroissement proportionnel du taux d'alphabétisation dans la langue étrangère, ce qui explique pourquoi la vernacularisation est surtout favorisée par les habitudes linguistiques des masses d'individus semilettrés. Telle est la situation qui prévaut dans la plupart des sociétés

${ }^{1}$ Nous nous abstiendrons autant que possible de porter un jugement de valeur sur la qualité de la langue vernacularisée. 
postcoloniales où les langues des anciennes métropoles servent de langues d'administration, d'enseignement, d'échange public en situation formelle et informelle, voire de langues véhiculaires. Ces langues varient selon le passé colonial des pays : anglais dans les anciennes colonies britanniques comme le Nigéria, le Ghana ou l'Ouganda, français et anglais au Cameroun, français dans les pays de l'ex-Afrique occidentale et équatoriale française (Sénégal, Bénin, Gabon, Congo, etc.), portugais en Angola ou au Mozambique, espagnol en Guinée Équatoriale. La superposition de ces langues étrangères aux vernaculaires locaux est à l'origine des variétés régionales du superstrat, lesquelles vont d'une forme standard locale à un pidgin (Dumont, 1990) lourdement tributaire du substrat. La vernacularisation est l'émergence du mésolecte qui se situe entre les deux pôles de ce continuum. Plusieurs études ont été consacrées aux variétés vernacularisées de ces langues en Afrique ${ }^{2}$, en particulier le français et l'anglais qui sont de loin les plus répandues. Mais à notre connaissance ces travaux ont surtout consisté en des descriptions d'ordre lexical et phonologique, et ont rarement porté sur le traitement de ces variétés dans un contexte traductologique. Nous nous proposons d'aborder quelques-uns des problèmes pratiques et thériques auxquels est confronté le traducteur des écrits non conformes à la norme des langues française et anglaise ${ }^{3}$. Nous nous appuierons pour ce faire sur les textes dits pragmatiques (Delisle, 1980).

Les textes pragmatiques (rapports, communiqués, motions et requêtes diverses) dont il est question ici proviennent d'une grande diversité de sources et leur traitement ne se prête pas aisément à la systématisation. Nous n'évoquerons que les difficultés qui nous semblent

2 Cf. par exemple pour le français l'Inventaire des particularités lexicales du français en Afrique noire (ACCT, 1975), ainsi que les ouvrages de Manessy $(1979,1984)$ ou Dumont (1990); pour l'anglais, les études portent en général sur les variétés sous-régionales comme le *West African Standard English* ou sur le Pidgin English (Dwyer, 1967; Todd, 1984; Menang, 1979; Simo Bopda, 1991).

${ }^{3}$ La plupart des exemples cités et des situations évoquées dans le texte viennent de notre propre expérience comme traducteur professionnel en Afrique subsaharienne, où nous avons travaille sur des textes en langue vernacularisée de divers pays dont le Cameroun, le Niger, le Nigéria, le Burkina Faso et la Côte d'Ivoire. 
les plus pertinentes et les plus généralisables. Loin de prétendre à l'exhaustivité, notre réflexion vise surtout à souligner quelques questions et points précis sur le thème évoqué par le titre. Dans la quasi-totalité des anciennes colonies, l'urbanisation, l'exode rural, les forts taux d'abandon scolaire, la médiocrité des sources de maintien et de renforcement du «bon usagew, le phénomène de désalphabétisation ${ }^{4}$, la pression culturelle de l'Occident, et les politiques linguistiques officielles, ont tous contribué à la création d'une masse d'individus semi-lettrés qui utilisent quotidiennement une variété non conforme à la norme de la langue officielle. On pourrait assimiler les écrits de cette masse à ceux des analphabètes des sociétés industrialisées fortement alphabétisées. Mais du point de vue de la traduction, un tel rapprochement ne saurait être poussé trop loin pour diverses raisons dont les suivantes : dans la plupart des pays occidentaux, les analphabètes ont très souvent pour langue maternelle une variété de la langue officielle et nationale; en plus, il existe ici un fond culturel commun à tous, au-delà des classes sociales et des degrés d'instruction; enfin, les dialectes et autres langues non officielles ont généralement fait l'objet d'études plus ou moins étendues, ce qui peut contribuer en principe à atténuer l'ampleur du problème des interférences ou tout au moins à le poser en des termes différents.

La plupart des études ayant trait à l'évaluation de la qualité des traductions pragmatiques font relativement peu de place à la qualité des textes de départ. En portant toute l'attention sur le produit final du processus traductionnel, on tient en général pour acquis que dans le texte source, l'auteur a respecté les normes grammaticales et stylistiques de la langue de départ. Une telle présupposition est certainement valable pour la plupart des textes produits dans les sociétés où le rédacteur, très souvent instruit, écrit dans sa langue maternelle. Mais dans notre contexte, seuls les écrits émanant de l'élite instruite (par définition minoritaire) sont susceptibles de remplir l'exigence de qualité. La traduction des textes produits par la majorité de la population, qui ne maîtrise pas la langue officielle, présente des difficultés attribuables à plusieurs facteurs, dont la

${ }^{4} \mathrm{Ce}$ terme renvoie à la perte de l'aptitude à communiquer efficacement dans une langue acquise de manière formelle. La désalphabétisation se manifeste essentiellement par l'oubli des règles d'orthographe et de grammaire, la contraction du vocabulaire, et l'érosion des compétencess relatives à la lecture et à l'expression au-delà du registre familier. 
méconnaissance des règles grammaticales et stylistiques de la langue, l'ignorance de la terminologie ou du jargon approprié, la traduction littérale d'expressions idiomatiques de la langue maternelle, le recours à des stratégies de communication propres à un contexte culturel traditionnel. Ces textes accusent par ailleurs des interférences provenant de deux principales sources : la variété locale du superstrat et la langue maternelle du rédacteur. Les interférences se manifestent particulièrement aux niveaux lexico-sémantique, phonologique et idiomatique, et elles nuisent à la compréhension et à la traduction du message original.

Au niveau lexico-sémantique, il n'est pas rare de rencontrer des néologismes locaux obtenus par dérivation ou par déformation du superstrat, ainsi que des termes empruntés directement de la langue maternelle de l'auteur ou d'un véhiculaire régional. L'observation ${ }^{5}$ révèle que le nombre d'emprunts est d'autant plus important que la langue véhiculaire représente une variété non standard de la langue officielle utilisée dans le texte. La raison en est probablement que la proximité apparente des deux codes en présence induit facilement en erreur le locuteur semi-lettré. Ainsi, des mots du Pidgin English se glissent dans les textes rédigés en anglais, tandis que ceux de ce qu'on pourrait appeler le "pidgin-français» se retrouvent dans les textes écrits en français. Quand ces termes proviennent du superstrat, leur interprétation est rendue plus complexe par le fait qu'ils ont généralement subi des modifications de sens, voire des transferts de catégorie grammaticale, comme l'illustrent les exemples suivants en Pidgin English ouest-africain :

1) la forme anglaise book renvoie à tout document écrit - qu'il s'agisse d'une seule feuille ou d'une encyclopédie -, ainsi qu'à l'ensemble des connaissances livresques (comme dans Mark know book : Marc est instruit)

2) (to) suffer est à la fois un verbe comme en anglais, et un substantif (My suffer go finish : ma souffrance/misère va prendre fin)

3) l'adjectif big sert aussi de verbe (This pikin de/di big : cet enfant grandit)

4) l'adjectif hungry est souvent utilisé comme un substantif (hungry

5 Cf. R. Mopoho 1994. 
de/di do we : nous mourons de faim) ou un verbe (Wuna de/di hungry? : avez-vous faim?)

On observe une situation similaire en français sub-saharien où de nombreux mots ont êté dérivés des formes existantes du français international (hexagonal) :

5) cadeauter signifie offrir un cadeau

6) garcerie désigne une maison close (où l'on trouve des "garces*)

7) enceinter veut dire rendre enceinte.

D'autres mots subissent des transferts ou des extensions de sens :

8) passer un examen signifie le réussir, et non pas tout simplement le subir

9) être luisant c'est avoir bonne mine, resplendir de santé

10) descendre veut dire arrêter de travailler (pour une période ou pour la journte)

11) le mot remède fournit un exemple d'extension sémantique : en plus de son sens initial, il renvoie également à toute substance nocive (le raticide est le remède de la souris) ou à un fétiche (on lui a fait le remède : il a été ensorcelé ou envouté).

Alors que la dérivation s'effectue sur des éléments de la langue étrangère, l'extension de sens (telle qu'illustrée en 11) est souvent le résultat d'un calque ou d'une traduction litterale d'expressions de la langue matemelle du locuteur. Bien que de nombreuses études se soient intéressées à ces phénomènes, peu d'africanismes de forme ou de sens sont entrés dans un dictionnaire général de la langue étrangère $e^{6}$. L'une des raisons en serait que ces néologismes sont fortement régionalisés, à cause en partie de l'histoire, des langues locales, et de leur vitalité qui n'est pas égale partout.

${ }^{6}$ Par exemple, le récent ouvrage de P. Krop (1995) Tu fais l'avion par terre a justement pour sous-titre «Dictionnaire franco-africain $m$, et les termes qu'il renferme, bien qu'ils soient souvent en usage depuis plusieurs décennies, ne sont jamais apparus ni dans le Petit Robert ni dans le Petit Larousse. 
Tous ces facteurs compliquent la tâche du traducteur qui est obligé, s'il ne maîtrise pas la variété vernacularisée de la langue officielle, de deviner le sens à partir du contexte.

Habituellement, les interférences phonologiques ne sont pas aisément attestables à l'écrit. Mais dans les textes produits par des personnes semi-lettrées, ces interférences mènent à des déviances sur le plan graphique. Ce phénomène est particulièrement important en anglais où l'on trouve des exemples fréquents de confusion entre des paires minimales comme match et march, robber et rubber, bag et back, soup et soap, three et tree, foot et food, etc. Le contexte permet généralement de découvrir ces erreurs et de les corriger dans la traduction.

Pour revenir aux interférences lexicales, les individus semilettrés ont tendance à introduire dans leurs textes des mots empruntés directement à leur langue matemelle. Il s'agit souvent de termes désignant des objets ou des concepts spécifiques à la culture locale, et pour lesquels l'auteur ne connaîtrait pas d'equivalents dans la langue officielle ou la variêté standard. Les exemples suivants illustrent ce genre de situation :

12) The matter will be taken before the ardo ${ }^{7}$

13) The Tekumbeng' women marched to the governor's office

14) The conversation was heard in a mbuh" parlor

15) The visitors brought a fehkeng ${ }^{10}$ leaf as a sign of peace.

Le traducteur qui n'a aucune connaissance de la culture ou de la langue maternelle de l'auteur du texte ne peut se fier entièrement au contexte

7 Chef traditionnel dans une communaute foulbe.

${ }^{a}$ Société secrète de femmes dans la plupart des groupes ethniques de la province du Nord-Ouest au Cameroun.

${ }^{9}$ Boisson alcoolisée de distillation locale.

${ }^{10}$ Branchage servant de symbole de paix. 
pour trouver le sens du mot «étrangem. La démarche la plus fructueuse consisterait à se renseigner auprès d'un locuteur natif.

II serait facile et tentant de limiter le phénomène de la vernacularisation à sa dimension lexicale, laquelle se manifeste sous la forme d'un langage imagé, kexotiquen, voire humoristique pour certains. $S^{\prime}$ 'il est vrai qu'un vocabulaire $\alpha$ singulier» est plus susceptible de frapper l'imagination du grand public, il est indéniable que la vernacularisation comporte aussi une dimension subtile dont les effets se prêtent moins facilement (par rapport à la dimension lexicale) à l'analyse et à la généralisation. Il s'agit des interférences idiomatiques, stylistiques et culturelles. Le problème qu'entraînent ces interférences est particulièrement évident quand les auteurs (semi-lettrés) écrivent dans une langue étrangère tout en se conformant aux codes d'interaction verbale et sociale de leur communauté ethnique d'origine. Les interférences idiomatiques prennent la forme de la traduction littérale d'expressions et de collocations de la langue maternelle, comme dans ${ }^{11}$ :

16) pleurer son corps : donner (toute) la mesure de ses talents

17) entrer dans l'ail de quelqu'un : s'en prendre à lui, généralement à tort

18) arrêter le vol : se plaindre d'avoir été volé.

Tout comme dans le cas des mots indigènes, l'interprétation des calques de ce genre nécessite une bonne connaissance de la langue maternelle de l'auteur.

En ce qui concerne les interférences culturelles, elles ont surtout trait à la stratégie d'argumentation adoptée par l'auteur. Par exemple, un candidat postulant un emploi évitera dans sa demande d'insister ouvertement sur ses qualités et ses expériences pertinentes, parce que dans sa culture une telle action trahirait un manque d'humilité. De nombreuses langues africaines regorgent de dictons et de maximes comme celle ciaprès qui stigmatisent tout comportement autolouangeur par lequel un individu vante ses propres qualités ou s'adresse des éloges : «Quand on a

"Langues d'origine : bamiléké (16 et 17) et bamoun (18). 
mis un beau chapeau, on ne le montre pas du doigt en disant "regardez comme mon chapeau est beau"; on laisse à d'autres - qui voient de loin à quoi le chapeau ressemble sur la tête - le soin de l'appréciero's'. Autre manifestation des interférences culturelles: les sujets tabous dans la culture de l'auteur sont abordés de manière indirecte, l'auteur recourant surtout à l'allusion et au sous-entendu ${ }^{13}$; en outre, lorsque le problème abordé est jugé sérieux, l'auteur s'abstient d'utiliser un langage clair et direct qui, dans son esprit, ne pourrait que contribuer à banaliser le sujet. De manière générale, de par la tradition, les sujets importants ou délicats sont laissés à ceux qui savent «bien parlers, c'est-à-dire qui maîtrisent l'art de l'expression tacite, où sont privilégiés le proverbe, la maxime, la parabole, la métaphore, la répétition habile comme moyen d'insistance, l'adage, les images, et de nombreux autres moyens discursifs du même genre. Le rédacteur laisse au lecteur-destinataire le soin de décoder le message pour le comprendre, ce qui amène le traducteur à lire constamment entre les lignes pour exprimer la totalité du message.

Dans une telle façon de procéder on pourrait voir la manifestation d'une attitude ou d'un état d'esprit propre aux locuteurs des langues «orales» qui n'ont pas de tradition d'écriture. Il s'agirait cependant moins d'une spécificité linguistico-culturelle que d'une stratégie particulière de communication. $\grave{A}$ preuve, la démarche discursive susévoquée présente des similarités avec celle qu'adoptent les locuteurs du japonais, langue qui a pourtant une longue tradition d'écriture. Comparant le japonais et l'anglais, Wakabayashi relève que :

Certain stylistic characteristics of Japanese are at variance with the ideals of good English - e.g. the emphasis on phatic language, the tolerance for repetitiousness and verbosity, and the high frequency of vague self-effacing expressions, hyperbole and rhetorical questions. (1990, pp. 26-27)

${ }^{12}$ Dicton de l'ouest du Cameroun.

${ }^{13}$ Il va sans dire que les tabous linguistiques peuvent varier considérablement d'une société africaine à l'autre, tout comme par rapport à l'Occident : les ceuvres de l'écrivain Chinua Achebe montrent que chez les Ibos du Nigéria un bon nombre de proverbes font référence aux parties intimes, à l'excrétion, aux infirmités, etc., dont l'évocation directe sous d'autres cieux serait contraire aux règles de la bienséance. 
Elle note par ailleurs (1991) que dans la stratégie de communication, le locuteur du japonais croit en la supériorité de l'argumentation intuitive sur l'argumentation logique. Elle conclut avec Hinds (1987, p. 143) que :

[...] whereas in English the writer is primarily responsible for effective communication, in Japanese the burden rests with the reader [...] in a reader-responsible language there is greater tolerance for ambiguity and imprecision.

Le problème de l'interprétation du message implicite est exacerbé par la grande diversité linguistique qui caractérise la plupart des sociétés africaines postcoloniales. Par exemple, la Côte d'Ivoire et le Cameroun, avec respectivement plus de 150 et 240 groupes ethnolinguistiques distincts, sont parmi les pays les plus linguistiquement hétérogènes au monde. En général, ici, il existe rarement une aculture nationalen homogène que partagent tous les citoyens, y compris l'auteur de l'original et le traducteur. Ce demier doit fréquemment travailler sur des textes dont le fond culturel lui est pour ainsi dire étranger. Il arrive souvent que les proverbes et adages, à l'instar des collocations, soient traduits littéralement de la langue maternelle de l'auteur. Le traducteur est alors contraint de solliciter laide d'un locuteur de la langue en question. Mais lorsque l'auteur a procédé à une traduction tout à fait compréhensible du proverbe dans la langue étrangère, la tâche du trađucteur peut s'en trouver allégée : il ne lui reste plus qu'à décider s'il veut conserver (et donc traduire) la version de l'auteur ou recourir à l'application d'un «filtre culturel» (House, 1977) dans la langue cible, comme dans l'exemple suivant :

19) Quand celui qui est dans la dèche tend un piège, il y prend son propre chien

Adaptation en anglais : it never rains but it pours (un malbeur n'arrive jamais seul)

Chez les personnes semi-lettrées qui sont en grande partie responsables du phénomène de vernacularisation, la non-maîtrise des normes de la langue étrangère a pour corollaire une connaissance souvent superficielle de la culture de cette langue. Des lacunes culturelles se manifestent dans leurs écrits et doivent nécessairement être comblées par le traducteur. La phrase suivante contient un exemple type de ce genre de lacunes: 
20) The mallam was sitting on the mat with some bibles in front of him $^{14}$

L'auteur du texte d'où est tirée cette phrase croit vraisemblablement que le mot mallam est familier aux locuteurs de l'anglais, ou tout au moins au destinataire original, et que sa signification est évidente. L'analyse montre toutefois que ce terme est non seulement étranger à l'anglais international ${ }^{15}$, mais qu'il a en outre au moins trois acceptions en Afrique sub-saharienne : d'une part, il désigne un titre conféré à un homme qui connaît les saintes écritures islamiques, se distingue par sa foi et peut officier lors des cérémonies religieuses; d'autre part, il renvoie à un guérisseur qui puise sa science dans le Coran; enfin, au sens péjoratif il désigne un envouteur ou un charlatan de religion musulmane (ou originaire d'une région à prédominance islamique) qui prétend détenir des pouvoirs de divination et de guérison. Encore une fois, seul le contexte peut permettre dans chaque cas de déterminer l'acception appropriée. La même phrase révèle également que pour l'auteur, le mot bible sert à désigner tout document à caractère religieux. Il est peu probable qu'un musulman, quelle que soit l'acception retenue pour le terme mallam, puisse posséder et étaler des «bibles" sur sa natte au vu et au su de tous, surtout dans un environnement où l'cecuménisme n'est pas spécialement à la mode. On peut valablement avancer qu'il s'agit plutôt de recueils coraniques.

Pour réexprimer la totalité du message du texte en langue vernacularisée, il s'avère donc indispensable non seulement de maîtriser les normes de la langue source, comme le suggère Newmark (1980), mais aussi de connaître la langue maternelle de l'auteur et sa culture, ainsi que de repérer et de combler les lacunes culturelles que le texte pourrait laisser apparâtre. Au bout d'une telle démarche se pose l'inévitable question de l'évaluation de la qualité du texte traduit. Nous aborderons cette question

\footnotetext{
${ }^{14}$ Phrase extraite d'un texte destiné à la traduction au Cameroun.
}

${ }^{15}$ Mallam est vraisemblablement une adaptation locale de l'arabe morâbit, «moinesoldat*; il serait intéressant de noter que ce terme a été adopté dans l'anglais africain, tandis que dans les pays francophones l'équivalent marabout est plus usité. 
en nous limitant à un réexamen de la pertinence de deux critères traditionnels, à savoir ceux de la fidélité et de l'équivalence fonctionnelle.

La notion de fidélité n'est pas entendue ici au sens ancien de reproduction rigoureuse et exclusive de la forme de l'original. Nous n'avons pas par ailleurs l'intention de réouvrir le sempiternel débat sur l'opposition entre la forme et le fond, qui est par ailleurs dénoncé comme suit par Steiner :

[...] from Ciceto to Saint Jerome until the present, the debate over the extent and quality of reproductive fidelity to be achieved by the translator has been philosophically naive or fictive. It has postulated a semantic polarity of "word" and "sense", and then argued over the optimal use of the space "between them". (1992, p. 292)

La fidélité pour nous consiste à préserver à la fois la forme et le fond du message, lesquels sont aussi indissociables que «le recto et le verso d'une feuille [...] et contribuent l'un et l'autre au sens global du message et a l'impression cognitive et affective qu'il laisse sur les lecteurs» (Delisle, 1980).

En principe, la fidélité peut être assurée, entre autres, par l'établissement d'une équivalence fonctionnelle ou sémantico-pragmatique (House, 1977) entre les textes de départ et d'arrivée. Lorsque dans l'original les normes grammaticales, stylistiques et idiomatiques de la langue de départ sont respectées, il se pose relativement moins de problèmes de compréhension, et l'exercice d'interprétation des idées peut se dérouler de manière plus ou moins objective. En conséquence, il peut être aisé de vérifier la fidélité générale du texte traduit. Mais lorsque par rapport aux normes de la langue de départ le texte à traduire présente des écarts du genre susévoqué, les contours de la forme étant moins précis, il devient plus difficile de saisir le fond. Plus le texte source est difforme, plus le processus traductionnel est subjectif. Les ouvrages de référence (unilingues ou multilingues) dont les entrées servent normalement au repérage des équivalences s'avèrent de peu d'utilité. Le traducteur du texte écrit dans une langue vernacularisée se trouve pratiquement contraint de faire abstraction du sens premier des unités graphiques qui constituent la forme du texte source. Il évolue dans une zone grise où il existe peu de correspondance entre le mot écrit et l'intention de l'auteur. Quand il a finalement saisi la globalité du message de l'original, il doit le reformuler, 
tout en gardant à l'esprit le fait qu'il n'est pas complètement libre de recréer à sa guise le texte source, car malgré ses lacunes, ce dernier limite son interprétation et lui permet ainsi ade dépasser les vagues intuitions impressionnistes" (Delisle, 1980). Parallelement, il doit s'efforcer de maintenir le ton général du texte, c'est-d̀-dire, selon le cas, savoir faire ressortir la menace, l'imploration, l'éloge, l'urgence, l'impatience, etc. exprimés par l'auteur du message original. Pour utiliser une image mille fois évoquée, le traducteur du texte vernacularisé apparaît comme un équilibriste soumis constamment a deux forces antagonistes : la soustraduction, que pourrait entraîner une interprétation superficielle de l'original, et la surtraduction, qui découlerait de la tendance à aller au-delà du propos de l'auteur. Pour reprendre Newmark (1980), la traduction ici exige wa degree of creative tension between fantasy and common sensew. On pourrait relever, non sans raison, que ce dilemme caractérise toute activité de traduction. Steiner (1992) note par exemple que dans toute compréhension il y a une interprétation active, et que même l'énoncé le plus littéral comporte dans son appréhension une dimension herméneutique. Toutefois, nous croyons que tout est question de degré d'interprétation. Il nous semble que le besoin et l'ampleur de l'interprétation paraissent plus grands dans le processus de traduction des textes écrits dans la variété vernacularisée d'une langue étrangère.

Nous ne saurions évoquer la forme sans parler du style. Le rédacteur semi-lettré qui est à l'origine du texte vernacularisé ignore très souvent les conventions relatives au style de présentation des textes officiels. Il incombe donc au traducteur de récrire le texte en se conformant aux formules habituellement utilisées dans chaque contexte. En s'imposant un tel cadre restrictif, il réduit les risques d'embellissement que l'on court en traduisant un texte qui de toute évidence nécessite des "améliorationsn. Dans une correspondance officielle rédigée à la manière d'une lettre personnelle, les modifications stylistiques qu'apporte le traducteur peuvent porter entre autres sur l'utilisation des formules consacrées d'introduction, de développement et de conclusion, sur l'indication du nom, du titre, de la formule d'adresse approprice (excellence, honorable, majesté...) et de l'adresse officielle du destinataire, ainsi que sur la spécification et la mise en relief de l'objet de la lettre. Cette adaptation aux normes stylistiques de la langue d'arrivée est indispensable pour assurer une communication efficace. 
Le concept de style tel qu'évoqué ci-avant donne une idée de l'influence que le destinataire peut exercer directement ou indirectement sur le processus traductionnel. Newmark (1980) souligne que dans la traduction des textes non conformes à la norme de la langue (comme dans toutes les traductions, pourrait-on ajouter), il est très important de savoir à qui est destiné le produit final. Ả ce sujet, Hewson et Martin (1991) déplorent $\mathrm{l}^{\prime}$ aabsence totalen du destinataire dans la theorie de la traduction. À notre avis cependant, le problème n'est pas tant celui de l'absence du destinataire que du caractère insignifiant du rôle qu'on lui attribue. En effet, le destinataire, que l'on évoque très souvent, est invariablement décrit comme un acteur passif auquel le traducteur doit *adapter le message d'origine. Hewson et Martin (1991) font remarquer à juste titre que dans la pratique le destinataire, qui est en général le demandeur ${ }^{16}$ de la traduction (translation initiator), tend à jouer un rôle capital dans le processus traductionnel, car par ses consignes (translation orders) il influence considérablement le produit final de l'opération traduisante. L'existence de nombreux lexiques maison et de guides de rédaction particuliers attestent largement de la réalité de ce fait. Il convient toutefois de préciser que le destinataire n'est pas toujours le demandeur : l'éditeur qui établit et octroie le contrat de traduction d'un ouvrage est le demandeur, tandis que le lecteur potentiel dans la langue d'arrivée est le destinataire. Les textes pragmatiques traduits dans notre contexte sont généralement destinés aux autorités publiques. Ils doivent souvent, conformément aux procédures administratives usuelles, passer par des destinataires intermédiaires (les bureaucrates subaltemes du destinataire final) qui ont la réputation - largement méritée - d'être tatillons. Les consignes sont habituellement implicites mais elles n'en restent pas moins contraignantes : la suite réservée à une requête dépend de sa clarté et de sa précision, et le traducteur sait qu'à toute étape de l' aétuden du dossier, celui-ci peut être rejeté sous divers prétextes, dont le moindre n'est pas le avice de formen.

De ce qui précède, la conclusion que l'on peut tirer semble être qu'en traduction de textes pragmatiques, la forme n'est pertinente, c'est-àdire digne d'être reproduite, que si elle est perçue d'une part comme étant correcte aux plans grammatical, stylistique et idiomatique, et d'autre part

${ }^{16}$ Dans le jargon professionnel au Canada, on parle aussi de donneur d'ouvrage. 
comme contribuant à l'expression de la totalité du message. Cette double condition n'étant pas remplie pour les textes écrits dans une langue et un style déviant de la norme, le traducteur peut faire abstraction de la forme initiale. Il se donne pour objectif primordial l'établissement d'une équivalence idéique (Delisle, 1980) entre les deux textes. En d'autres termes, pour paraphraser Dryden ${ }^{17}$, le traducteur réécrit l'original comme l'aurait fait l'auteur s'il maîtrisait la langue d'arrivée et le protocole de rédaction applicable à son genre de texte.

Dans la traduction des textes pragmatiques, en particulier dans des situations de vernacularisation, les difficultés relatives à l'interprétation et aux divers choix d'adaptation telles qu'évoqués dans le présent article sous-tendent un problème fondamental, à savoir celui de la limite et de la pertinence des interventions du traducteur sur le texte original. Doit-il ameliorer ce dernier par le biais de la traduction afin de le rendre accessible au public cible, ou lui faut-il se contenter de le reproduire textuellement, au risque d'aboutir à une version inintelligible? Dans quelle mesure peut-il jouer au rédacteur-censeur et purifier le texte avant sa livraison aux destinataires? Faut-il qu'il s'assure que la "qualité" du texte est la mềme dans la langue source et la langue cible? Le texte amélioré est-il encore une traduction ou une création parallèle (la covert version de House, 1977)? Il est aisé de constater que chez les spécialistes les avis sont partagés sur toutes ces questions. Le débat est loin d'être clos. En attendant, il peut être utile de remarquer que l'on ne devrait jamais perdre de vue ni les conditions de rédaction de l'original ni l'intention de l'auteur - en supposant que le texte la révèle clairement. Si la déviation par rapport à la norme est un acte conscient de l'auteur, comme dans certains textes littéraires, les passages non conformes à la norme contribuent, par leur forme même, à exprimer la totalité du message. Dans ces conditions, *corrigem la langue de l'original reviendrait à amputer le message. Par contre, si les déviations sont clairement des fautes attribuables au manque de maîtrise de la langue telle qu'elle se manifeste dans les écrits des individus semi-lettrés, on peut légitimement estimer,

${ }^{17}$ Pour Dryden, la traduction doit refléter l'original tel qu'il aurait été conçu par l'auteur lui-même dans la langue d'arrivée. De sa traduction de Virgile, il dit : a[...] I have endeavoured to make Virgil speak such English as he would himself have spoken, if he had been born in England, and in this present ageo (cité par Steiner, 1992, p. 269). 
selon nous, que le texte gagnerait à être reproduit dans une langue recevable.

\section{Dalbousie University}

\section{Références}

DELISLE, Jean (1980). L'analyse du discours comme méthode de traduction. Ottawa, University of Ottawa Press.

DUMONT, Pierre (1990). Le français langue africaine. Paris, LHarmattan.

DWYER, David (1967). An Introduction to West African Pidgin English. Michigan, Michigan University Press.

GENTZLER, Edwin (1993). Comtemporary Translation Theories. London/New York, Routledge.

HALL, R.A. Jr. (1966). Pidgin and Creole Languages. Ithaca, Cornell University Press.

HEWSON, Lance and Jacky MARTIN (1991). Redefining Translation. The Variational Approach. London/New York, Routledge.

HINDS, J. (1987). «Reader Versus Writer Responsibility: A New Typology”, U. Connor and R. Kaplan (Eds.), Writing Across Languages: Analysis of L2 Texts. Redding (Mass.), Addison Wesley, pp. 141-152.

HOUSE, Juliane (1977). A Model for Translation Quality Assessment. Tubingen, Gunter Narr.

MANESSY, Gabriel (1979). «Le français en Afrique noire : faits et hypothèses", Albert WALDMAN (dir.), Le français hors de France. Paris, Honoré Champion. 
MANESSY, Gabriel et Paul WALD (1984). Le francais en Afrique noire -tel qu'on le parle, tel qu'on le dit. Paris, L'Harmattan.

MENANG, T. (1979). Trends in Cameroon Pidgin English Lexicology. A Study of Anglophone Speech. Thèse de M.A., inédite, University of Yaounde.

MOPOHO, Raymond (1987). Terminologie des études sur la traduction. Mémoire de maîtrise, inédit, Université de Montréal.

(1994). Multilinguisme et communication au Cameroun. Études sociolinguistiques. Thèse de Ph.D., inédite, Université de Montréal.

NEWMARK, Peter (1981). Approaches to Translation. Oxford, Pergamon Press.

York/London/Toronto, Prenticehall.

(1988). A Textbook of Translation. New SIMO BOPDA, A. (1991), «Does Pronunciation Matter?», English Teaching Forum, $29,4$.

STEINER, George (1992). After Babel. Oxford/New York, Oxford University Press.

TODD, Loreto (1984). Modern Englishes. Oxford, Basil Blackwell.

WAKABAYASHI, Judy (1991). "Translation Between Unrelated Languages and Cultures, as Illustrated by Japanese-English Translation*. Meta, 36, 2/3, pp. 414-423.

RÉSUMÉ : Vernacularisation et traduction des textes pragmatiques en Afrique - La traduction des textes comportant des lacunes d'ordre grammatical, lexical, stylistique ou idiomatique présente habituellement des difficultés particulières, lesquelles sont amplifiées lorsqu'elles sont attribuables à la vemacularisation d'une langue étrangère. Dans les sociétés postcoloniales, l'absence ou la non-disponibilité des études 
linguistiques sur la plupart des langues locales rend ardue l'analyse des interférences entre ces dernières et les langues officielles étrangères. Cette situation, ajoutée à la grande diversité ethnolinguistique ambiante, ne facilite pas l'interprétation des textes produits par les personnes semilettrées. Le traducteur de ces textes se présente davantage comme un rédacteur qui, à partir de l'idée globale qui se dégage de l'original, conçoit et produit un texte répondant aux normes de la langue cible. L'évaluation d'un tel travail ne peut se faire qu'en comparant la finalité des deux textes.

\begin{abstract}
Vernacularisation and Translation of Pragmatic Texts in Africa - Written texts with grammatical, lexical, stylistic or idiomatic gaps usually present specific challenges in translation. These challenges are amplified when they stem from the vernacularization of a foreign language. In postcolonial settings, the analysis of interference between local and foreign languages is hindered by the absence or unavailability of linguistic studies related to local languages. When added to the prevailing ethnolinguistic diversity, this situation renders the interpretation of writings by semi-literate individuals more arduous. The translator appears to be more of a (re)writer who relies on the general idea as expressed in the original to produce a text that conforms to the norms of the target language. Assessing the result of such a process can only be based on a comparison of the aim or function of each text involved.
\end{abstract}

Raymond Mopoho : Département de français, Dalhousie University, Halifax, N.S. B3H 3J5, Téléphone : (902) 494-2018, Télécopie : (902) 494-1626 